\title{
Brain Imaging for Autism Diagnosis
}

\author{
An undergraduate student is working to develop an imaging analysis \\ technique for the speedy diagnosis of autism spectrum disorders, which \\ could allow clinicians to start treatment earlier than is currently possible. \\ By Katherine Wright
}

$\square$ hysics is the science of the universal: finding common rules that govern the behaviors of disparate systems. But medical physicists have a different approach, as many diseases call for customized-rather than blanket-treatment plans. That is where a new technique for characterizing structures in the brain might come in handy, hopes Shannon Brindle, a final-year undergraduate physics major working in the lab of Rasha Makkia at the University of Mary Washington, Virginia. For example, she envisions that advanced brain imaging analysis techniques could help clinicians verify - at a younger age than currently possible-whether a child has an autism spectrum disorder,
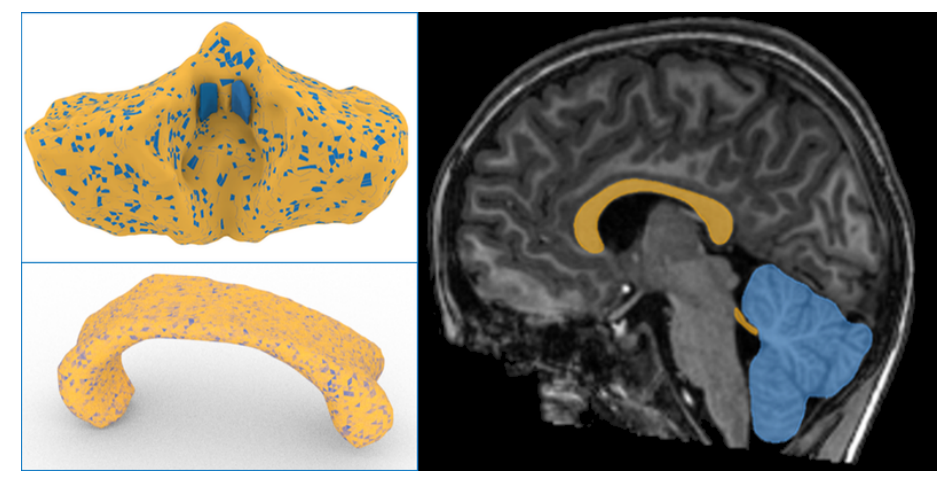

Reconstructions of the cerebellum (top left) and the corpus callosum (bottom left) obtained from a magnetic resonance imaging (MRI) scan (right) of a child diagnosed with an autistic disorder spectrum. The yellow structure in the MRI corresponds to the corpus callosum and the blue structure to the cerebellum. Credit: S. Brindle, C. Saben, R. Makkia/University of Mary Washington pinpoint its severity, and then better tailor interventions to that child's individual needs. Brindle discussed her findings in a talk at this year's March Meeting of the American Physical Society.

One in 54 children in the US receives an autism diagnosis each year, 5 times more than the number of children who develop cancer. But no blood or imaging tests exist for the disorder. Rather, the disorder, which develops in early childhood, is diagnosed by studying a child's ability to communicate and interact with other people. That means it can take time for a family to receive a formal diagnosis, which can delay treatment and leave parents fretting for answers.

Brindle, who has three cousins with autism, understands these difficulties all too well. So when it came to picking out her undergraduate research project, she was instantly drawn to one that had the potential to help in fixing the problem. "This issue is very close to my heart," she says. "This project was a very family oriented one."

Brindle, in tandem with another student, Clark Saban, helped in developing a computational technique that can simultaneously extract multiple structures from 3D magnetic resonance imaging brain scans, something not possible with other methods. The technique isolates the structures and then renders them with graphical representations called NURBS, which capture and maintain the topological intricacies of the structures more precisely than other methods. Brindle used her technique to characterize two structures: the cerebellum, which is at the back of the brain and is involved in the control of motor functions, and the corpus callosum, a c-shaped bundle of nerve fibers that connects the brain's right and left sides. Changes in 
the sizes of neurons within these structures and the functioning of these brain regions has been linked to autism. The structures are also easily identifiable, making them promising targets for diagnosing the disease, Brindle says.

To test her method, Brindle applied it to images of the brains of two 8-year-old boys who had been diagnosed with autism, and to one 8-year-old boy who hadn't. Brindle notes that there is a higher prevalence of autism in boys than in girls, a factor that played a role in the image choice. Also, as an undergraduate she has limited access to scans. "These were the ones that were available to me right now," she says.

Analyzing the structures, Brindle made two observations. First, she found that the cerebella were larger for the boys with autism, while the corpus callosa were smaller, something expected from other autism studies. Second, the right "stalk," known as a peduncle, that connects the cerebellum to the rest of the brain, was smaller than the left peduncle in the boys with autism. The opposite was true for the other boy. While the measurements appear to be meaningful, Makkia notes that they have yet to undergo a thorough vetting both in her lab or by peer review. She also notes that in published results for girls with autism, the left peduncle is the bigger one. A radiologist has however confirmed that Brindle's technique has correctly isolated the structures, Makkia says.

With preliminary results on only three patients, it is obviously too early to claim that the observations have clinical significance. But perhaps the ingenuity of an undergrad student could provide a fresh approach to a long-standing problem.

Brindle hopes to add more patients to the study when she starts graduate school. She also envisions extending her method to monitor other diseases in children, such as cancerous brain tumors. The hope is to provide tools that could help clinicians develop more personalized treatment plans for a variety of conditions. "If I were a parent, I would want to see doctors use a computer model to make calculations specific to my child to make sure that they were kept safe," she says.

Katherine Wright is the Deputy Editor of Physics. 